\title{
Short-Chain Fatty Acid Profile in the Colon of Newborn Piglets Using Fecal Water Analysis
}

\author{
ROBERT D. MURRAY, H. JUHLING MCCLUNG, B ULYSSES K. LI, AND ANTON AILABOUNI
}

Department of Pediatrics, Ohio State University, Columbus Children's Hospital, Columbus, Ohio 43205

\begin{abstract}
Short-chain fatty acid production and assimilation is unlikely to occur at significant levels in the newborn because the colon at birth is sterile, and only gradually acquires an anaerobic flora. This study profiled short-chain fatty acid levels in the colon lumen over the initial 21 days of life. Fecal samples were removed surgically from the cecum, right, and left colon from 36 York piglets, 0-21 days of life. Samples were subjected to in vitro dialysis and centrifugation methods to quantitate fecal water short-chain fatty acids, electrolytes, osmolality, and pH. A three-way analysis of variance examined piglet age, colon site of fecal samples, and method of fecal water analysis, for each variable. No differences were found between techniques of fecal water collection. Newborns showed production of short-chain fatty acids as early as the 1st day of life in limited amounts. Levels were stable between days 5 and 14, and then abruptly accumulated in the lumen. Acetate was predominant early, with propionate and butyrate responsible for late peaks. The production and assimilation of short-chain fatty acids was nearly complete proximal to the left colon. Age and colon site showed significant interactions for each fatty acid $(p<$ 0.001 ). The combined osmolar contributions of short-chain fatty acids and electrolytes accounted completely for the luminal osmolality after the 2 nd wk of life. Previously there was an "osmolar gap" suggesting that lactose or its breakdown products were present in the lumen and were being removed by pathways other than through short-chain fatty acid production. (Pediatr Res 22:720-724, 1987)
\end{abstract}

\section{Abbreviations}

\author{
SCFA, short-chain fatty acids \\ CEN, centrifugation \\ DIA, in vitro dialysis \\ POST-DIA, postdialysis centrifugation \\ ANOVA, analysis of variance \\ ip, intraperitoneal
}

The passage of carbohydrates across the ileocecal valve is a normal phenomenon throughout life. A metabolic pathway for carbohydrate digestion in the colon has been well established (1). In the anaerobic environment of the colon, fecal organisms ferment carbohydrate to gasses $\left(\mathrm{H}_{2}, \mathrm{CO} 2, \mathrm{CH} 4\right)$ and SCFA (acetate, butyrate, and propionate). The presence of SCFA is critical to the normal physiology of the colon. SCFA absorption from the lumen of the colon is rapid and efficient, and their

Received April 29, 1987; accepted August 11, 1987.

Address requests for reprints to Robert D. Murray, M.D., Gastroenterology Division, Children's Hospital, 700 Children's Drive, Columbus, $\mathrm{OH} 43205$.

Supported by the Children's Hospital Research Foundation and a grant from the Bremer Foundation. uptake enhances sodium and water absorption from the lumen, thereby preventing osmotic diarrhea (2-4).

Studies have substantiated bacterial fermentation of carbohydrates in premature $(5,6)$ and term newborns (7) on a formula diet, in breast-fed infants (8), in infants taking a mixed diet (9), as well as in older children (10). In newborns, measured intestinal lactase activity is inadequate to account for the digestion of a normal lactose load (approximately $2 \mathrm{~g} / \mathrm{kg} /$ feeding) (11). Calculations from in vitro data and at least one clinical study have suggested that up to two-thirds of the ingested lactose may be assimilated from the colon $(5,6,11)$. While it has been assumed that the same colon fermentation pathway used in adults accounts for lactose assimilation in newborns, this supposition is unlikely. The colon at birth is sterile and only gradually acquires the requisite anaerobic flora and milieu that allow production of SCFA in large quantities (12). The purpose of the present study was to profile SCFA appearance in the fecal water contents in newborn piglets fed sow milk over the initial 3 wk of life. Our studies demonstrate that SCFA are not present in substantial quantities until the $3 \mathrm{rd}$ wk of life, and that before that time there exists an "osmolar gap" between the osmolar contributions from SCFA and electrolytes and the measured total osmolality.

\section{METHODS}

Fecal samples. Newborn piglets of the York breed were used. Littermates were housed with the sow in isolation from the remainder of the swine herd until the day of study to assure a normal field flora. Piglets were exclusively suckled, restricted from access to solids, and exempted from iron and antibiotic injections. Piglets, aged $0-21$ days, ranged in weight from 1.2 to $5.7 \mathrm{~kg}$ on the day of study. No attempt was made to regulate either the feeding schedule or the amount of milk suckled per feed.

Surgery was performed within four hours of procurement. Animals were anesthesized with Nembutol ( $30 \mathrm{mg} / \mathrm{kg}$, ip), intubated, and ventilated. The $\mathrm{pH}, \mathrm{PCO}_{2}, \mathrm{PO}_{2}$, bicarbonate, and $\mathrm{O}_{2}$ saturation were monitored using a $\mathrm{pH}$ blood gas analyzer $(158$, Corning Medical and Scientific, Medfield, MA). Body temperature was maintained by a heat lamp in conjunction with a heating pad placed under the piglet. A temperature probe in the rectum regulated core temperature between $37-39^{\circ} \mathrm{C}$. A V-shaped incision was made with a Bovie unit such that a bloodless exposure of the entire colon was possible. Warmed saline $\left(40^{\circ} \mathrm{C}\right)$ was applied to the colon for heating and wetting of the surface.

A small incision was made with the Bovie unit in the cecum tip and the contents gently milked into a vial and placed on dry ice. A similar step was used to obtain samples from the right colon approximately $20 \mathrm{~cm}$ distal to the cecal incision, prior to the colon coil. A left colon sample was taken just distal to the coil. When possible, two separate samples of more than $1 \mathrm{~g}$ in size were obtained from each location, and results averaged. Samples were each weighed and homogenized in distilled water, with $100 \mu \mathrm{l}$ of $\mathrm{HgCl}_{2}, 0.06 \mathrm{M}$, added to halt bacterial metabolic 
activity. After samples were collected the piglets were euthanized using pentobarbital injection.

Volatility of SCFA. To study the recovery of SCFA after processing the stool, three weighed fecal specimens were divided. diluted with distilled water, and homogenized after the addition of $0.2 \mu \mathrm{Ci}$ of ${ }^{14}(\mathrm{C}$-acetate. Two dilutions were compared per sample, 1:4 and 1:10. Samples were centrifuged at $18.000 \mathrm{rpm}$ for $20 \mathrm{~min}$ at $4^{\circ}($ and the supernatant recovered. Supernatant was counted and the recovery of ${ }^{14} \mathrm{C}$ recorded per stool weight. The whole pellet, free of supernatant. was then counted to determine residual ${ }^{14} \mathrm{C}$.

Comparison of fecal water techniques. A comparison of fecal water collection techniques was performed on 32 luminal samples from 13 piglets, four in the ()- 10 7-day age group, and nine in the 14- to 21-day age group. For these studies each sample of fecal homogenate was diluted $1: 10$ in distilled water and was subjected to two methods for obtaining fecal water: a centrifugation technique, and a technique of in vitro dialysis (13). Samples undergoing CEN were centrifuged at 18,000 rpm for $20 \mathrm{~min}$ at $4^{\circ} \mathrm{C}$, and the supernatant immediately aspirated for analysis. Homogenate undergoing DI $\wedge$ was poured into a small beaker. $\wedge$ 6-cm bag of cellulose dialysis tubing. mol wt cutoff $12-16.000$ (Spectropore, Spectrum Medical, Los Angeles. CA), was filled with a $10 \%$ Dextran solution, $60-90,000)$ daltons (Sigma Chemical Co., St. Louis, MO), immersed into the fecal homogenate, and then allowed to dialyze to completion over $24 \mathrm{~h}$ at $4^{\circ} \mathrm{C}$, following the method of Vernia et al. (13). The bag was then removed, its contents aspirated, and immediately analyzed. The fecal homogenate remaining in the beaker after dialysis (POSTDIA) was then centrifuged at $18,000 \mathrm{rpm}$ for $20 \mathrm{~min}$ and the supernatant compared to that from both the prior CEN and dialysis techniques for pH, osmolality, electrolytes, and SCFA composition.

SCFA profile. When the initial 32 samples were analyzed, no differences were demonstrated between the CEN, DIA, and POST-DIA techniques. Thereafter, the CEN method was used exclusively to analyze 111 stool samples from 36 piglets spanning ages (0-21 days in order to closely profile the SCFA and osmolality content of the fecal water over the course of the neonatal period.

SCFA osmolalit: The presence of osmoles in stool above the osmolar contribution of SCFA and electrolytes would suggest that other constituents are present in fecal water. This difference-the "osmolar gap"-is represented by the following: Osmolar gap $=$ total luminal osmolality (mosmol/liter) $-($ SCFA mosmol/liter + electrolytes mosmol/liter). Because the measured osmolality of acetate, propionate, and butyrate was less than the concentration on a mmol/liter basis, an estimate of the osmolar contribution from the three SCFA was experimentally determined. Over a range of concentrations from $250-1000 \mathrm{mmol} /$ liter the osmolality was 86,85 , and $81.5 \%$ of the total $\mathrm{mmol} /$ liter concentration of acetate, propionate, and butyrate, respectively. The mean osmolality of a mixture of the three SCFA was $84 \%$ of the concentration, a factor that was then applied to the measured total SCFA concentration as determined by gas chromatograph, to estimate the osmolar contribution of SCFA in fecal water.

Analytical methods. Sodium and potassium contents were analyzed by flame photometry (II.343 Flame Photometer. Instrument Laboratory Inc.. Lexington, MA). Chloride and bicarbonate were analyzed by potentiometric titration $(6616$ Beckman C1-CO 2 Analyzer, Beckman Instrument Inc., Cedar Grove. NJ). Osmolality was measured by freczing point depression (Advanced Micro-Osmometer 3 MO, Advanced Instruments Inc.. Needham Heights, MA) and the $\mathrm{pH}$ measured by $\mathrm{pH}$ Meter (Accumet pH Meter 810, Allied Fisher Scientific. Pittsburgh, PA). For the analysis of acetate, propionate and butyrate, a Hewlett-Packard gas chromatograph equipped with a flame ionization detector (HP 5580A Hewlett-Packard, Palo Alto, CA) and a level four integrator terminal was employed. The aqueous samples were injected on a $1 / 5$ inch $\times 6$ foot nickel 200 column packed with Gaschrom 220. an 80/100 mesh, porous polymer packing of low polarity (Alltech Assoc. Inc.. Applied Science labs. Deerfield. II.). Ethyl butyrate was used as an internal standard. added just prior to chromatographic analysis.

Statistics. The initial 32 fecal samples were compared by threeway ANOVA. to compare the means of multiple groups (14). The three variables included the techniques of obtaining fecal water (CEN versus DIA versus: POST-DIA), piglet age groups (0-7 versus 14-21 days), and the colon locations of each sample (cecum versus right versus left colon). Multiple 3-way analyses were performed using $\mathrm{pH}$. osmolality, individual clectrolytes. and each SCFA as individual dependent variables. The lechniques for obtaining fecal water were further compared by correlation coefficients. On the 111 samples obtained exclusively by the centrifugation technique, a two-way ANOVA was then performed, comparing piglet age versus colon location, with total SCFA as the dependent variable. The two-way ANOVA was then repeated with the cecal data excluded to assess the contributions of the right and left colon to SCFA production. independently.

\section{RESLITS}

No loss of ${ }^{14} \mathrm{C}$ - acetate by volatilization was found in samples undergoing homogenization and centrifugation to obtain fecal water. This was true either when diluted $1: 10$ (mean $=99.5 \%$ recovery, range $96-101 \%)$ or $1: 4$ (mean $=102 \%$ recovery, range $101-103 \%$. The remaining pellet accounted for less than $5 \%$ of the original counts in each instance.

Comparison of fecal water methods. Data from the initial 32 stools are summarized in Table 1 . Within the 3-way ANOVA, the method used to obtain fecal water was not a significant interacting variable either with piglet age or with the colon site from which the stool was obtained. The technique did not demonstrate a main effect in explaining differences found between group means. Correlation coefficients $(r)$. used to compare CEN versus DIA and DIA versus POST-DIA groups, are displayed in Table 1 and suggest that the methods are similar. Dialysis was complete after $24 \mathrm{~h}$, evidenced by the fact that centrifugation of the stool remaining after dialysis (POST-DIA) reproduced the data obtained by aspirating the dialysis bag. Within the 3-way ANOVA, besides a comparison of fecal water methods. age and colon site interactions were also analyzed. $\wedge$ ge demonstrated a main effect with each dependent variable (electrolytes. SCFA. osmolality. and $\mathrm{pH}$ ), while colon site had a main effect only with sodium and potassium $(p<0.01)$. The interaction of age and colon site for chloride. bicarbonate, $\mathrm{pH}$, and osmolality was not significant. For each SCFA, however, age group. colon site. and their interaction were all highly significant variables $(p<0.0001)$.

SCF1 profile. The luminal appearance of SCFA was profiled in fecal water collected exclusively by the centrifugation technique from 111 luminal samples. as illustrated in Figure I. SCFA could be found in measurable amounts as early as day 1. Luminal levels in the cecum remained stable between days 5 and 14 and then abruptly accumulated thereafter. In the period following day 14 , levels in the cecum were approximately three times greater than those in the right colon. and right colon values in turn were nearly three times greater than those in the left colon. Prior to day 14. the presence of SCFA in the cecum was limited. Cecum and right colon values were almost equal during this time. A two-way ANOVA comparing the colon site vs age for the total SCFA curve confirmed the interaction of the two variables $(p<0.001)$. When the ANOVA was repeated with cecal data excluded, the interaction remained highly significant $(p<0.001)$.

The individual SCFA that made up the curve did not fluctuate in a fixed ratio (Fig. 2.1). All three SCF $\triangle$ appeared in the stool on day 1, with acetate predominant. Over wk 1 and 2. acetate remained the most prominent fatty acid as all three gradually 
Table 1. Comparison of fecal water techniques*

\begin{tabular}{|c|c|c|c|c|c|c|c|c|c|}
\hline & $\mathrm{Na}$ & $\mathrm{K}$ & $\mathrm{Cl}$ & $\mathrm{HCO}_{3}$ & OSM & $\mathrm{pH}$ & A & $\mathrm{P}$ & $\mathrm{B}$ \\
\hline CEN & 51 & 74 & 26 & 2.0 & 378 & 6.9 & 64 & 77 & 46 \\
\hline$\pm \mathrm{SEM}$ & 6.5 & 3.8 & 2.1 & 0.1 & 8.9 & 0.0 & 5.7 & 11.2 & 8.2 \\
\hline DIA & 46 & 66 & 25 & 2.3 & 371 & 7.0 & 64 & 77 & 47 \\
\hline$\pm \mathrm{SEM}$ & 6.3 & 4.1 & 2.0 & 0.1 & 8.2 & 0.0 & 5.7 & 11.2 & 8.2 \\
\hline POST-DIA & 47 & 66 & 26 & 2.0 & 368 & 6.9 & 62 & 77 & 46 \\
\hline $\begin{array}{l} \pm \text { SEM } \\
\text { CEN vs DIA }\end{array}$ & 6.3 & 4.5 & 1.6 & 0.1 & 8.6 & 0.0 & 5.7 & 11.0 & 8.2 \\
\hline $\begin{array}{l}(r) \\
\text { DIA } v s \\
\text { POST-DIA }\end{array}$ & 0.98 & 0.84 & 0.96 & 0.71 & 0.96 & 0.92 & 0.98 & 0.99 & 0.99 \\
\hline$(r)$ & 0.97 & 0.93 & 0.88 & 0.73 & 0.97 & 0.96 & 0.98 & 0.99 & 0.99 \\
\hline
\end{tabular}

* Comparison of fecal water techniques for analysis of electrolytes, SCFA, osmolality, and pH, based on 32 stool specimens from 13 piglets. Values from CEN, DIA, and POST-DIA of the remaining fecal homogenate are represented as mean \pm SEM. Correlation coefficients $(r)$ between CEN and DIA and between DIA and POST-DIA values are represented.

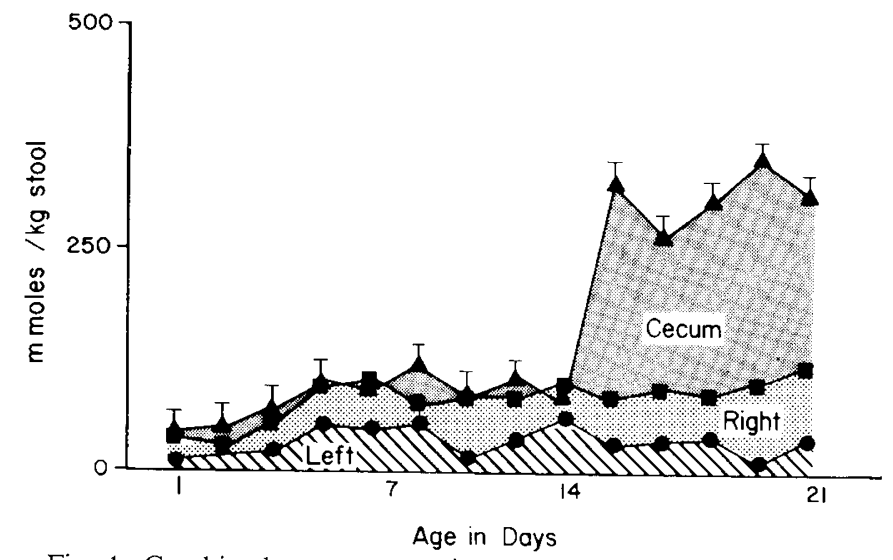

Fig. 1. Combined acetate, propionate, and butyrate mean values separated by colon site over the first $3 \mathrm{wk}$ of life. Interactions between age and colon site were significant by two-way ANOVA with and without cecum data included $(p<0.001)$.

rose. Propionate and butyrate showed an abrupt acceleration in luminal concentrations after day 14 . These two SCFA accounted for almost all the late peak in the total SCFA profile. The semilog curve (Fig. $2 B$ ) demonstrates the rapid shifts in luminal levels of each SCFA over the first 3 wk of life, corresponding to the steepness of slope. Of the three, butyrate showed the most pronounced variation in luminal concentration.

The osmolality in fecal samples consists of a number of particles present in solution. Contributions to the solute pool come from various ions and minerals, from partially digested dietary nutrients, from gastrointestinal secretions and mucin, from bacteria, and from the products of bacterial fermentation of undigested nutrients. In Figure 3, the total osmolality in luminal samples from the cecum is compared with the summed contributions from ions and SCFA. Following day 16, SCFA and ions together completely accounted for the total osmolality in the luminal stream. Before day 16, however, a "gap" existed of luminal osmoles that remained unaccounted for by summing electrolyte and SCFA osmolar contributions; this totaled as much as 75 mosmol.

\section{DISCUSSION}

Unlike ruminants, adult humans on a normal dietary intake of carbohydrates do not rely extensively on the colon for energy absorption (15-17). However, newborns taking the relatively high lactose load in breast milk or milk-based infant formula may rely on the colon as the site of digestion for as much as $65 \%$ of the ingested carbohydrates, closely mimicking the situation in ruminant animals (6). Our results suggest that in the first $2 \mathrm{wk}$ of life, lactose in the newborn colon is not metabolized through
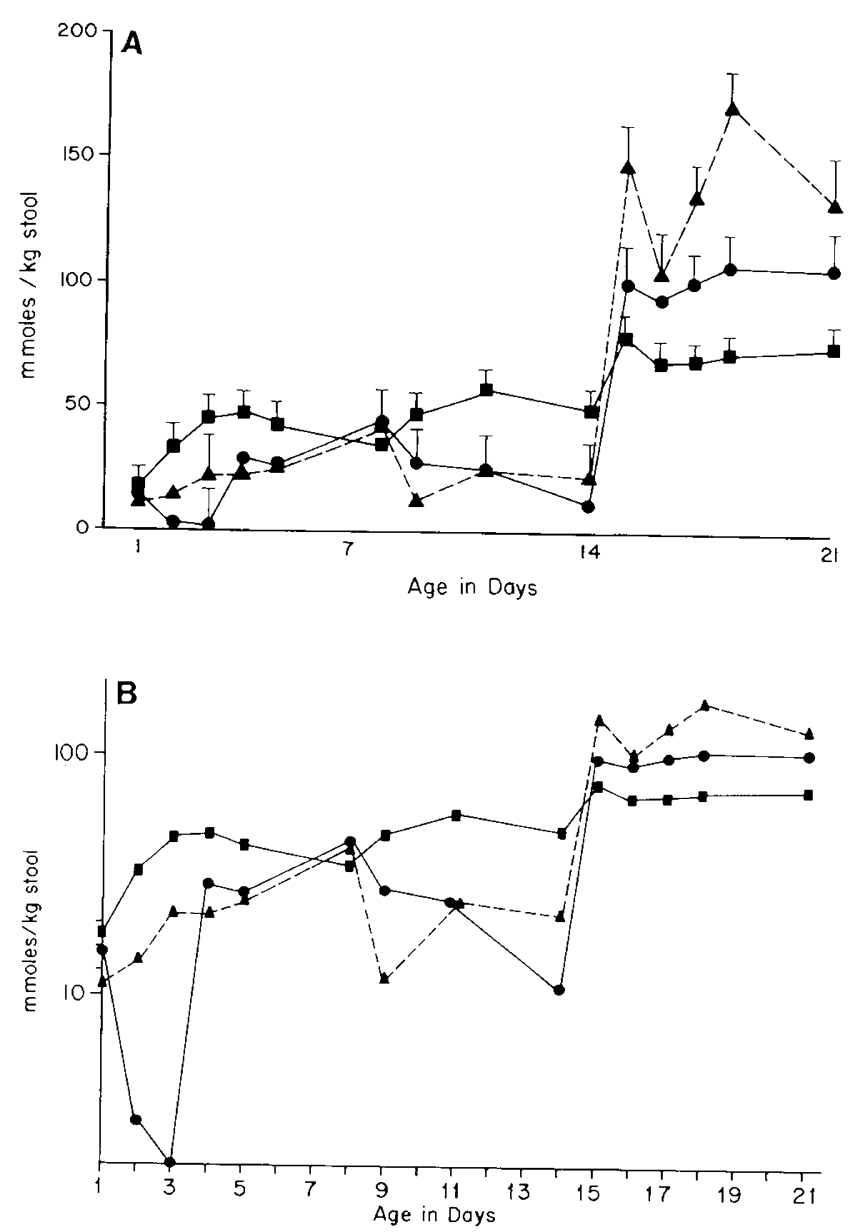

Fig. 2. A, changes in individual SCFA in the cecum over the course of the first 3 wk of life. Mean acetate $(\boldsymbol{\square})$, propionate $(\boldsymbol{\Lambda})$, and butyrate $(\bullet)$ values were represented. $B$, the same data on a semilogarithmic scale illustrates the rate of change of the three SCFA over time. Age is significant $(p<0.001)$ for each SCFA.

the same pathways as in older infants and adults; i.e. through pathways that result in the production of SCFA. A profile of the total SCFA in fecal water demonstrates that levels rise abruptly after the 2 nd wk of life, at a time when the anaerobic flora in the colon achieves a population similar to that in the adult (12). The osmolality from the SCFA fully accounts for the difference between the total measured osmolality and the osmolality generated from electrolytes in feces following day 14-16 of life. Prior to that time an osmolar gap exists of unidentified particles in fecal water that may represent either lactose, its direct breakdown 


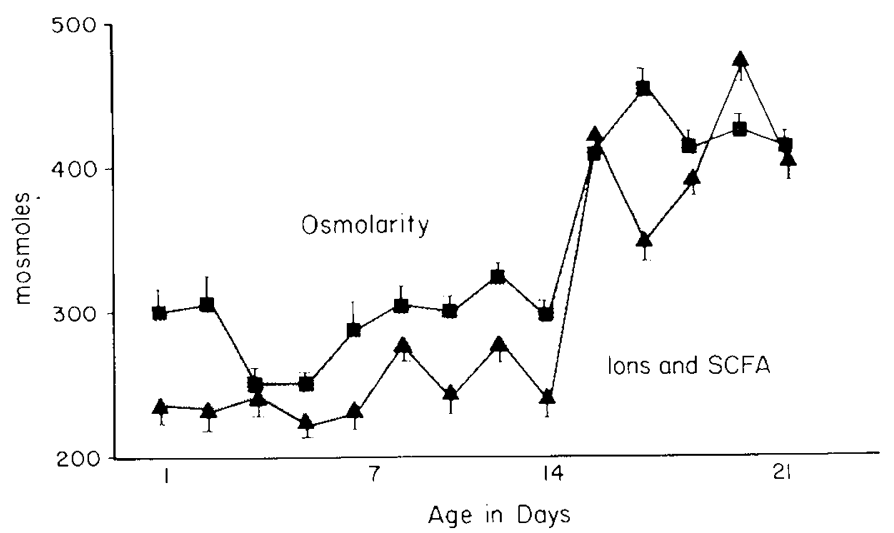

Fïg. 3. The difference between total luminal osmolality in the cecum as measured by freering point depression and the summed osmolat contributions from electrolyte ions $\left(\mathrm{Na}^{+}, \mathrm{K}^{+}, \mathrm{Cl}^{-}, \mathrm{HCO}_{3}\right)$ and SCFA over the study period. The disparity, the osmolar gap, is represented in the shaded area of the curve.

products, glucose or galactose, or fermentation products of metabolism from the mixed anacrobic and acrobic flora present in the colon during the first weeks after birth.

Pigs have a remarkable similarity to humans with regard to cardiovascular, bone and mineral, nutrient, and gastrointestinal physiology $(18,19)$. Close correlation has been shown between the response of the newborn piglet and that of the human neonate to gastrointestinal infection (20). Although the lactose load in sow milk averages $6 \mathrm{~g} / \mathrm{dl}$, very similar to that in human breast milk, carbohydrate handling by the piglet is probably not completely analogous to the human neonate (21). Iactase activity in humans rises in the weeks preceding and just following birth (11). In contrast, intestinal lactase activity is highest at birth in the piglet, and falls progressively over the 1 st month of life (22. 23). This may be one factor in the rise in SCF $\triangle$ in the cecum. although the fall in the enzyme is gradual over the 3-4 wh after birth in piglets. The piglet model, therefore is likely to underestimate the amount of carbohydrate that is handled by the colon in human newborns. The osmolar gap noted in the first 2 wh of life in the piglet would likely be substantially greater in humans. especially in prematures below $36 \mathrm{wk}$ gestational age when lactase levels are negligible. Based on this information, humans probably rely to an even greater extent on colon pathways other than SCr:A absorption to retrieve lactose energy in the first weeks of life.

Several technical issues were addressed in this study. Potential volatilization and loss of SCFA during processing was originally a concern. ${ }^{14} \mathrm{C}$-acetate, the smallest SCFA and the one most likely to volatilize, when added to fecal samples prior to homogenization and centrifugation, was completely recovered. irrespective of dilution. The pH of the fecal homogenates remained above pl1 6.4, well above the pK of all three SCFA. 4.75-4.87 at $25^{\circ} \mathrm{C}$. All three techniques of collecting fecal water had almost identical values for all three SCFA (Table 1), yet were handled much differently, also suggesting that volatilization of SCFA did not occur. ${ }^{14} \mathrm{C}$-acetate was also used to examine whether a high percentage of extracellular water was trapped within the pellet during centrifugation. Recovery of ${ }^{14} \mathrm{C}$-acetate was essentially $100 \%$. Various methodologies have been applied to the analysis of fecal water. Vernia e' al. (13), compared in vitro dialysis against ultrafiltration and found less than $5 \%$ difference between the two when analyzing exchangable ions, SCFA, pH. and osmolality. In the study presented herein. in vitro dialysis was in turn compared with centrifugation, and similar results were obtained. The chromatographic methods chosen allowed direct injection of fecal water samples. This methodology was first validated by Henkel (24) using a similar packing material to measure SCFA in biologic samples.
An ideal method to evaluate fecal contents would be one which estimates the chemical composition of the stool without altering it. Methods that incinerate or chemically digest the stool have proven inaceurate to evaluate exchangeable ions. Various methods have been utilized to obtain the fecal water directly: chemical precipitation atter dilution of the fecal contents, cither in vive or in litro (13). However, cach of these methods has drawbacks. While the method of ultracentrifugation gives an accurate sample of fecal water, its yeld from formed stool is small. Dialysis methods function by allowing a chemical equilibration across a membrane cither passing through the colon in rito, or placed in a stool sample in vitre until equilibration is complete. The study reported herein sought to evaluate samples from three locations in the colon. Results from the in ritro dialysis technique were compared to those from simple eentrifugation on stool which had been previously diluted. This dilution step adds an artifact to the osmolality by altering the activit! coefficients of the solutes in stool. which in turn raises the reported osmolality. This artifact is the principle criticism of any method of fecal water analysis that uses a dilution step. In order to generate sufficient fluid to perform the study. this artilact had to be accepted. All samples were treated identically. making comparisons within the study valid. However, comparisons of the absolute osmolality with literature values not using the dilution technique cannot be made.

Aurrichio et al. (11) used lactase digestive velocity in ritro to estimate the maximum lactose digestion that might oceur in the small bowel of newborns. Using his conservative measures. over a 24-h period no more than $15 \mathrm{~g}$ of lactose could be hydrolyed and assimilated in the small bowel by infants at 41 wh gestational age, an amount far below the $38 \mathrm{~g}$ ingested by an average 3-kg infant taking six $3-07$ feedings per day. Mact can and link (6) used breath hydrogen values in prematures as a measure of the amount of carbohydrate undergoing bacterial fermentation. By their estimates, up to two-thirds of the ingested lactose entered the colon. Prematures showed a linear increase in breath H. production over the first 3 wk. with $\mathrm{H}_{2}$ recovered from $100 \%$ of the infants only after the $3 \mathrm{rd}$ wh of life. Conversely, detectable losses of ${ }^{13} \mathrm{C}$-labeled lactose in stool occurred in less than half of the term infants studied, and in those infants only a mean of $3.3 \%$ of the ingested ${ }^{3}$ C-lactose dose was recovered (7). Similar results were found in stools of prematures $(5)$. The bacterial thora appear to be an important second enzyme system in newborms for the digestion of lactose that would otherwise be lost in feces.

The assumption that the metabolic pathway utilized by the fecal flora in newborns is similar to that in adults is not supported by this study. Bacterial acquisition in all mammals follows a well-defined sequence (12). The colon is initially sterile. Aerobic organisms initiate colonization and subsequently the redox potential falls. Modest numbers of several different alerobic and anaerobic species appear over the next several days as the lumen becomes increasingly anaerobic.

The metabolic products of a burgeoning mixed flora will necessarily be diverse. The levels of SCFA. which represent products from a variety of anaerobic bacterial metabolic pathways, are limited in the first 2 wh of life. There are two primary plateaus of SCFA over the first 3 wh of life. one at day 5 and the other at day 14 (Fig. 1). These two points closely parallel the initial colonization and later proliferation of anacrobic flora in the colon, respectively (12). The semilogarithmic chart in rigure $2 B$ is used to illustrate the rate of change of the individual SCFA. correlated with the steepness of the slope for each variable (25). The broad variations seen in the semilogarithmic representation suggest extensive changes in bacterial metabolic activity. Butyrate and propionate account for nearly all the sudden accumulation of SCFA after dav 14. likely tied to the fact that bacteria proliferate in vast numbers at this time, under increasingly favorable anaerobic luminal conditions. Read sequentially from cecum to right to left colon. Figure I suggests that the cecum and right colon remove nearly all SCFA from the fecal stream prior to 
entry into the left colon, which would indicate that analysis of SCFA in feces after passage through the entire large bowel does not reflect the total metabolic activity that occurred higher in the colon.

Bacterial acquisition may not be the only factor responsible for the distinctive SCFA profile seen in this study. The anaerobic milieu of the colon, as measured by the redox potential, becomes increasingly negative following day 14 (22). At the same time, the piglet intestinal tract grows at a remarkable rate, $0.8 \mathrm{~cm} / \mathrm{h}$ during the 1st wk of life (24). Additional cecal size and surface area would accommodate a greater fecal volume and a slower transit time, and thus increase bacterial number, favoring fermentation. The volume of feedings and the lactose load per day continually rise in piglets over this time and will add to the volume of SCFA produced. Finally, absorption of SCFA from the colon lumen may be more pronounced early in life.

Acknowledgments. The authors thank Darlene Achee and Stacey Harper for their expert assistance with the preparation of the graphs and the manuscript, John Hayes for his active participation in data analysis and presentation, and William C. MacLean for his frequent reviews of this project.

\section{REFERENCES}

1. Bond JH, Levitt MD 1976 Fate of soluble carbohydrate in the colon of rats and man. J Clin Invest 57:1158-1164

2. McNeil NI, Cummings JH, Jamew WPT 1978 Short chain fatty acid abosorption by the human large intestine. Gut 19:819-822

3. Sakata T, vonEngelhardt W 1983 Stimulatory effect of short chain fatty acids on the epithelial cell proliferation in rat large intestine. Comp Biochem Physiol 74A:459-462

4. Crump MH, Argenzio RA, Whipp SC 1980 Effects of acetate on absorption of solute and water from the pig colon. Am J Vet Res 11:1565-1568

5. MacLean WC Jr, Murray RD, Wong W, Klein PD 1984 Comparative absorption of glucose and lactose in prematures assessed with ${ }^{13} \mathrm{C}$-substrates. Pediatr Res 18:205A

6. MacLean WC, Fink BB 1980 Lactose malabsorption in premature infants: magnitude and clinical significance. J Pediatr 97:383-388

7. MacLean WC, Fink BB, Schoeller DA, Wong W, Klein PD 1983 Lactose assimilation by full-term infants: relation of (13C) and $\mathrm{H} 2$ breath tests with fecal (13C) excretion. Pediatr Res 17:629-633
8. Lifschitz CH, O'Brian Smith E, Garza C 1983 Delayed complete functional lactose sufficiency in breast-fed infants. J Pediatr Gastroenterol Nutr 2:478482

9. Barr RG, Hanley J, Patterson DK, Woodridge J 1984 Breath hydrogen excretion in normal newborn infants in response to usual feeding patterns: evidence for "functional lactase insufficiency" beyond the first month of life. J Pediatr 104:527-533

10. Solomons NW, Viteri F 1978 Development of an interval breath sampling hydrogen $\left(\mathrm{H}_{2}\right)$ breath test for carbohydrate malabsorption in children: evidence for a circadian pattern of breath $\mathrm{H}_{2}$ concentration. Pediatr Res 12:816823

11. Auricchio S, Rubino A, Murset G 1965 Intestinal glycosidase activities in the human embryo, fetus, and newborn. Pediatrics 35:944--954

12. Cooperstock MS, Zeed AJ 1983 Intestinal flora of infants. In: Henteges DJ (ed) Human Intestinal Flora in Health and Disease. Academic Press, New York, pp 79-99

13. Vernia P, Brewer RI, Bhaedinger A, Lattella G, Santoro ML 1984 Composition of fecal water: comparison of "in vitro" dialysis with ultrafiltration. Gastroenterology 86:1557-1561

14. Godfrey K 1985 Comparing the means of several groups. N Engl J Med 313:1450-1456

15. Wolin MJ, Miller TL 1983 Carbohydrate fermentation. In: Hentges DJ (ed) Human Intestinal Microflora in Health and Disease. Academic Press, New York, pp 148-150

16. Stephen AM, Haddad AC, Phillips SF 1983 Passage of carbohydrate into the colon: direct measurements in humans. Gastroenterology 85:589-595

17. Levitt MD 1983 Malabsorption of starch: a normal phenomenon. Gastroen terology 85:769-770

18. Dodds WJ 1982 The pig model for biomedical research. Fed Proc 41:247-256

19. Newport MJ, Henschel MJ 1984 Evaluation of the neonatal pig as a model for infant nutrition: Effects of different proportions of casein and whey protein in milk in nitrogen metabolism and composition of digesta in the stomach. in milk in nitrogen meta
Pediatr Res 18:658-662

20. Davidson GP, Gall DG, Petric M, Butler DG, Hamilton JR 1977 Human rotavirus enteritis induced in conventional piglets: intestinal structure and transport. J Clin Invest 60:1402-1409

21. Elliot RJ, Vander Noot GW, Gilbreath RL, Fisher H 1971 Effect of dietary protein level on composition changes in sow colostrum and milk. J Animal Sci 32:1128-1137

22. Hartman PA, Hays VW, Baker RO, Neagle LH, Catron DR 1961 Digestive enzyme development in the young pig. J Animal Sci 20:114-123

23. Dollar AM, Mitchell KG, Porter JWG 1957 The utilization of carbohydrates in the young pig. Nature 179:1299

24. Henkel HG 1971 Gas chromatographic analysis of low boiling fatty acids in biological media. J Chromatogr 58:201-207 25. Schmid CF 1986 Whatever happened to the semilogarithmic chart? Am Stat
$40: 238-244$

26. Smith MW 1976 Sodium transport by the newborn pig intestine: functional changes during the first few days of postnatal life. In: Robinson JWL (ed) Intestinal Ion Transport. MTP Press, Lancaster, pp 213-232 